\title{
A Simple RP-HPLC Method for the Determination of Cefdinir in Human Serum: Validation and Application in a Pharmacokinetic Study with Healthy Bangladeshi Male Volunteers
}

\author{
Golam Mortuza Shahed, Md. Ashik Ullah, Abdullah Al Maruf, Maizbha Uddin \\ Ahmed, Mohammad Safiqul Islam, Zebun Nahar and Abul Hasnat
}

Department of Clinical Pharmacy and Pharmacology, University of Dhaka, Dhaka-1000, Bangladesh

\begin{abstract}
In the present study, a simple RP-HPLC method with UV detection has been validated to determine cefdinir concentrations in human serum samples and applied to determine the pharmacokinetic parameters of cefdinir in healthy Bangladeshi male volunteers. The mobile phase consisting of a mixture of $0.2 \mathrm{M}$ sodium dihydrogen phosphate buffer ( $\mathrm{pH} 3.2 \pm 0.05$ adjusted with o-phosphoric acid) and methanol at a ratio of 70:30 (v/v), was pumped at a flow rate of $1.0 \mathrm{ml} / \mathrm{min}$ through the $\mathrm{C}_{18}$ column at room temperature and the chromatographic separation was monitored at a wavelength of $254 \mathrm{~nm}$ with a sensitivity of 0.0001 AUFS. Cefaclor was used as internal standard. The developed method was selective and linear for cefdinir concentrations ranging from 0.05 to 5 $\mu \mathrm{g} / \mathrm{ml}$ for serum samples. The lower limit of quantification was defined as the lowest concentration on the calibration curve $(0.05 \mu \mathrm{g} / \mathrm{ml})$ for which an acceptable accuracy of $111.60 \%$ and a precision of $7.65 \%$ were obtained, while the minimum detectable quantity of cefdinir was found to be $0.02 \mu \mathrm{g} / \mathrm{ml}$. The intra-day and inter-day coefficient of variation (CV) at $0.05 \mu \mathrm{g} / \mathrm{ml}$ were $7.65 \%$ and $9.72 \%$, respectively. The average recovery of cefdinir from serum was $96.43 \%$. Acceptable results were obtained during stability study. The mean $\mathrm{C}_{\max }$ of cefdinir was found to be $1.42 \pm 0.53 \mu \mathrm{g} / \mathrm{ml}$ attained at a mean $\mathrm{T}_{\max }$ of $3.81 \pm 0.96 \mathrm{hr}$. The mean elimination half-life was 2.03 hours. This method proved to be simple, accurate and precise for pharmacokinetic and bioequivalence studies of cefdinir.
\end{abstract}

Key words: Method validation, Cefdinir, Antibiotic, Pharmacokinetics, Bangladeshi Male volunteer.

\section{INTRODUCTION}

Cefdinir is a semi-synthetic, extended spectrum third generation cephalosporin found to be active against both gram-positive and gram-negative bacteria. Chemically it is known as [6R-[6a,7ß(Z)]]7-[[(2-amino-4-thiazolyl) hydroxyimino) acetyl] amino]-3-ethyl-8-oxo-5-Thia-1-azabicyclo-(4.2.0.)oct-2-one-2-carboxylic acid. ${ }^{1,2}$ Cefdinir exhibits its bactericidal activity by inhibiting cell wall synthesis. It is found to be stable in presence of some, but not all $\beta$-lactamase enzymes. As a result, many organisms resistant to penicillins and some cepholosporins are susceptible to cefdinir. ${ }^{3}$ Of several oral cephalosporins, cefdinir is recommended as an

Correspondence to: Abul Hasnat

Phone: +880-2-9661920-73 Extn. 8164; Fax: +880-2-8615583

E-mail: ahasnat99@yahoo.com

Dhaka Univ. J. Pharm. Sci. 10(2): 109-116, 2011 (December) alternative therapy for children with acute otitis media who have type 1 hypersensitivity to betalactamse. ${ }^{4}$ It is usually a well-tolerated antibiotic, with most adverse effects being mild and selflimiting. ${ }^{1,5,6}$ Following a single dose of $200 \mathrm{mg}$ cefdinir, $\mathrm{C}_{\max }, \mathrm{T}_{\max }$ and $\mathrm{AUC}_{0-12}( \pm \mathrm{SD})$ of cefdinir were found to be $1.45(0.32) \mu \mathrm{g} / \mathrm{ml}, 4 \mathrm{hr}$ (range $3-5$ hr) and 6.99 (1.6) $\mu \mathrm{g}-\mathrm{hr} / \mathrm{ml}$, respectively. ${ }^{7}$ Cefdinir does not undergo extensive metabolism and its activity is primarily due to the parent molecule itself. It is eliminated principally via renal excretion with a mean $( \pm \mathrm{SD})$ serum elimination half-life of 1.7 ( \pm $0.6)$ hrs. $^{7}$

Analytical methods employed for quantitative determination of drugs and their metabolites in biological fluids are the key determinants in generating reproducible and reliable data that in turn are used in the evaluation and interpretation of 
bioavailability, bioequivalence and pharmacokinetics. ${ }^{8}$ A few methods are available for analysis and assay of cefdinir from biological samples, but none of them is away from limitations. ${ }^{7,9}$ Hence, the objectives of the study were to develop and validate a HPLC method for the determination of cefdinir from serum samples with good resolution still having the desired sensitivity when applying to the validated method in a pharmacokinetic study of cefdinir in healthy Bangladeshi male volunteers.

\section{MATERIALS AND METHOD}

Materials. Cefdinir (97.9\% purity) and cefaclor (internal standard, 95.6\% purity) were kind gift from Eskayef Bangladesh Ltd., Dhaka, Bangladesh. Methanol (HPLC grade) was obtained from SigmaAldrich Laborchemikalien $\mathrm{GmbH}$ (Germany). Sodium dihydrogen phosphate, disodium hydrogen phosphate, sodium hydroxide and ortho-phosphoric acid were of analytical grade and were used without further purification.

Instrumentation. A Shimadzu (Kyoto, Japan) HPLC system was used in quantification of Cefdinir consisting of a SCL-10Avp system controller, two LC-8A pumps. Data acquisition was performed and processed using LC solution (Version 1.03 SP3, Kyoto, Japan) software running under Windows XP on a Pentium PC. Ultraviolet detection was achieved with a SPD-10 Avp UV-VIS detector (Shimadzu Corporation, Kyoto, Japan). A Milli-Q ${ }^{\circledR}$ (Millipore, France) water purification system was used to obtain the purified water for the HPLC analysis.

Chromatographic conditions. The chromatographic separation was achieved on a reversed phase $\mathrm{C}_{18}$ column (Nucleosil $\mathrm{C}_{18}: 5 \mu ; 4.6$ x $250 \mathrm{~mm}$; MACHEREY-NAGEL GmbH \& Co., Germany) using a mobile phase composed of $0.2 \mathrm{M}$ sodium dihydrogen phosphate buffer ( $\mathrm{pH} 3.2 \pm 0.05$ adjusted with o-phosphoric acid) and methanol at a ratio of $70: 30(\mathrm{v} / \mathrm{v})$. The flow rate was set at $1.0 \mathrm{ml} / \mathrm{min}$ and separation was performed at room temperature. The chromatogram was monitored at $254 \mathrm{~nm}$ with a sensitivity of 0.0001 AUFS. Quantification of cefdinir was done by plotting cefdinir to internal standard (cefaclor) peak area ratio as a function of cefdinir concentration. The method of analysis was validated under the principles of Good Laboratory Practice through the following parameters: linearity, precision (intra-assay and inter-assay), accuracy, limit of quantification (LOQ), validation of the dilution factor, specificity, stability, and recovery. ${ }^{10}$

Preparation of stock solutions. The diluent for standard preparations was prepared by dissolving $0.117 \mathrm{gm}$ of sodium dihydrogen phosphate and 0.142 gm of disodium hydrogen phosphate in water and the $\mathrm{pH}$ was adjusted to $7.0 \pm 0.05$ by $10 \%$ sodium hydroxide solution. Then the volume was made 100 $\mathrm{ml}$ by adding water followed by filtering through 0.2 $\mu \mathrm{m}$ nylon filter and was degassed before use. Stock solution of cefdinir was prepared at the concentration of $10 \mu \mathrm{g} / \mathrm{ml}$ in diluent. Cefaclor (internal standard) stock solution was prepared in diluent to have a concentration of $5.0 \mu \mathrm{g} / \mathrm{ml}$.

Preparation of calibration standards for serum sample assay. Calibration standards were prepared by adding required amount of cefdinir stock solution, $100 \mu \mathrm{l}$ of drug free serum (protein precipitated) and $100 \quad \mu \mathrm{l}$ of cefaclor (internal standard) solution $(5 \mu \mathrm{g} / \mathrm{ml})$ to the diluent to achieve the cefdinir concentrations of 5.0, 2.0, 1.0, 0.5, 0.2, $0.1,0.05 \mu \mathrm{g} / \mathrm{ml}$. These samples were analyzed by the HPLC for the construction of calibration curves (Figure 1) and for method validation. Calibration curve was constructed by plotting the peak area ratio of cefdinir to cefaclor against the concentration of cefdinir. Similarly quality control (QC) samples were prepared at concentrations of 5.0, 0.5 and $0.05 \mu \mathrm{g} / \mathrm{ml}$.

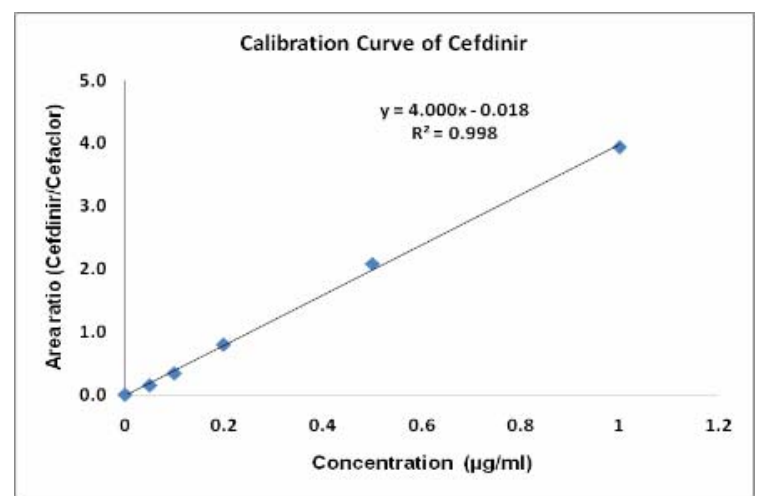

Figure 1. Calibration curve of cefdinir 
Preparation of serum sample and analysis. To $500 \mu \mathrm{l}$ of serum sample, $100 \mu \mathrm{l}$ of internal standard (cefaclor) solution $(5 \mu \mathrm{g} / \mathrm{ml})$ and $400 \mu \mathrm{l}$ of methanol were added. The mixture was vortexed for $15 \mathrm{sec}$ and then centrifuged at $10,000 \mathrm{rpm}$ for 5 minutes. The supernatant was transferred to disposable polypropylene tube and kept at $-80{ }^{\circ} \mathrm{C}$ until analysis. $20 \mu \mathrm{l}$ of the sample was injected into the column after filtering through $0.2 \mu$ syringe filter and analyzed by HPLC with UV detection.

\section{Method validation}

Specificity. The specificity of the method was established by analyzing blank sample, calibration sample spiked with cefdinir and internal standard (cefaclor) and processed volunteer's sample spiked with internal standard in serum. The retention times were confirmed for both cefdinir and cefaclor and the peak purity was evaluated.

Linearity and range. The linearity of the assay method was performed with seven point's calibration curve in serum. The slope and the intercept of the calibration graphs were calculated through least squares by weighing linear regression of drug to internal standard peak-area ratio and the concentration of cefdinir was studied over the range 0.05 to $5.0 \mu \mathrm{g} / \mathrm{ml}$ in serum. The standard curves were used to calculate concentrations of the analytes in unknown and QC samples from the measured peak area ratios.

Limit of quantification (LOQ) and limit of detection (LOD). The LOQ was estimated by analyzing samples with known amounts of cefdinir, at progressively lower concentrations. The LOQ was considered as the concentration level in which accuracy and precision were still better than $20 \%$. LOD is a parameter that provides the lowest concentration of analyte in a sample that can be detected, but not quantified, under the stated experimental conditions. The analyte concentration that produced a signal-to-noise ratio of 3:1 was accepted as the LOD. The analyte having the concentration lower than the LOQ was analyzed with progressively lower concentrations to determine the LOD. $^{8,11}$
Precision. The intra-assay (intra-day) and inter-assay (inter-day) variability of the method were assessed by analyzing quality control (QC) samples. The precision was expressed as relative standard deviation (RSD) or coefficient of variation (\%CV). The RSD to be determined at each concentration level should not exceed $15 \%$ for the method to be precise. $^{11}$

Accuracy. The accuracy was determined by standard addition method at different concentration levels of cefdinir. Different volumes of cefdinir were added to serum samples spiked with cefdinir and were analyzed by HPLC.

Extraction efficiency. Absolute recoveries of cefdinir at three QC levels were measured by assaying the samples as described above and comparing the peak areas of both cefdinir and internal standard. with those obtained from direct injection of the compounds dissolved in the supernatant of the processed blank serum.

\section{Application in pharmacokinetic study}

Volunteers. A total of eight healthy male volunteers were enrolled into the study; mean age $23.75 \pm 0.68$ years (range $19-30$ years); mean body weight, $71.43 \pm 7.9 \mathrm{~kg}$ (range $52-84 \mathrm{~kg}$ ); mean height, $1.71 \pm 0.09 \mathrm{~m}$ (range $1.61-1.83 \mathrm{~m}$ ) and mean body mass index (BMI), $21.92 \pm 2.27 \mathrm{~kg} / \mathrm{m}^{2}$ (range $18.16-26.14 \mathrm{~kg} / \mathrm{m}^{2}$ ). All the volunteers completed the study without any adverse effects.

All volunteers were examined to verify their healthy status; including medical history, vital sign measurements, electrocardiography (ECG), blood sample analysis (basic profile, complete blood cell count, bleeding time, clotting time, prothrombin time, viral serology), and urinalysis (sediment, drugs). Volunteers with relevant clinical, analytical, or ECG abnormalities were excluded from the trial. Additional exclusion criteria were as follows: smoking; history of alcohol or other drug abuse; consumption of any medication within one month prior to commencement of study, participation in a clinical trial in the 4 months before enrolment; history of clinically important illness or major surgery in the last 6 months; inability to relate to 
and/or cooperate with the investigators; medication allergy; illnesses or disorders that could affect the absorption, distribution, metabolism, and/or excretion of drugs (e.g., malabsorption, oedemas, renal and/or hepatic failure); a history of positive serology for hepatitis $\mathrm{B}$ or $\mathrm{C}$ (not due to immunization) or HIV; blood loss or donation in the 3 months before enrolment; blood or blood-derivative transfusion in the 6 months before enrolment; and excessive physical exercise in the 72 hours before enrolment. All eligible volunteers provided written consent to participate and they had right to withdraw from the study at any time without any obligation.

Study design. The protocol for the study was reviewed and approved by Bangladesh Medical Research Council (BMRC) and the study was conducted at the Department of Clinical Pharmacy and Pharmacology, Faculty of Pharmacy, University of Dhaka from January 2009 to June 2010. The study was conducted in accordance with the International Conference of Harmonization (ICH) guidelines for Good Clinical Practice (GCP) and in compliance with the Declaration of Helsinki and its further amendments. ${ }^{12,13}$ The study was a single-dose, randomized, open-label, one-period study. A single dose of $300 \mathrm{mg}$ of cefdinir capsule formulation $\left(\mathrm{CEDNIR}^{\circledR}\right.$, Eskayef Bangladesh Ltd., Dhaka, Bangladesh) was administered with $250 \mathrm{ml}$ of water after an overnight fasting. A standardized breakfast and lunch were given at 4 and 8 hours after drug administration, respectively. During the study period, the volunteers were under medical surveillance to report any adverse events. None of the volunteers vomited and no adverse effects were identified or reported.

Blood sampling. A $20-\mathrm{G} \times 1.25$-inch catheter (Vasofix ${ }^{\circledR}$ Braunüle ${ }^{\circledR}$, B.Braun Melsungen AG, Melsungen, Germany) was inserted into a suitable forearm vein and a $3 \mathrm{ml}$ of blood was withdrawn in each time of collection. Venous blood samples were obtained prior to dosing $(0 \mathrm{hr})$ and at $0.25,0.50,1.0$, $1.5,2.0,3.0,3.5,4.0,6.0,8.0$, and $12.0 \mathrm{hr}$ after administration of drug. The blood samples were kept in a dark place and then centrifuged at $1500 \mathrm{rpm}$ for 15 minutes at $25^{\circ} \mathrm{C}$. The separated serum was stored at $-80{ }^{\circ} \mathrm{C}$ until further analysis.

Bioanalysis. Cefdinir and cefaclor (internal standard) were extracted from serum samples by protein precipitation method using methanol. ${ }^{14}$ After protein precipitation, the supernatant was transferred to polypropylene tube and stored at $-80{ }^{\circ} \mathrm{C}$ until further analysis. $20 \mu \mathrm{l}$ of the sample was injected into the chromatographic system analyzed according to the method described above.

Pharmacokinetic and statistical analysis. Pharmacokinetic properties were calculated by a noncompartmental approach for the serum concentrations of cefdinir using software Kinetica (Version 4.4.1, Thermo Electron Corporation, UK). $\mathrm{C}_{\max }$ was estimated directly from observed concentrations, and $\mathrm{T}_{\max }$ as the corresponding time point at which $\mathrm{C}_{\max }$ occurred. $\mathrm{AUC}_{0-\mathrm{t}}$ was calculated by the linear trapezoidal method until the last measurable serum drug concentration, and $\mathrm{AUC}_{0-\infty}$ was calculated as $\mathrm{AUC}_{0-\infty}=\mathrm{AUC}_{0-\mathrm{t}}+\mathrm{C}_{\text {last }} / \mathrm{K}_{\mathrm{el}} \cdot \mathrm{k}_{\mathrm{el}}$ was the terminal elimination rate constant calculated by linear least square regression of the last three to four time points in the log concentration time profile and the terminal half-life was calculated by the following equation ${ }^{11}: \mathrm{t}_{1 / 2}=0.693 / k_{e l}$. The mean residence time (MRT) was calculated as:

$$
\mathrm{MRT}=\frac{\mathrm{AUMC}_{0-\infty}}{\mathrm{AUC}_{0-\infty}}
$$

\section{RESULTS AND DISCUSSION}

Using the optimized extraction method and chromatographic conditions, the HPLC method was evaluated in terms of specificity, linearity, limit of detection, limit of quantification, precision, accuracy, and recovery.

Selectivity and chromatography. Representative chromatograms are illustrated in Figure 2. These chromatograms include a processed blank sample, processed calibrator sample spiked with cefdinir, processed calibrator sample spiked with cefaclor and processed volunteer sample spiked with internal standard in serum. As illustrated in each of these chromatograms, the retention times of the cefdinir 
and cefaclor were approximately 5.6 and 6.3 minutes. cefdinir were completely resolved from one another The chromatograms showed that cefaclor and without any interference (Figure 2).

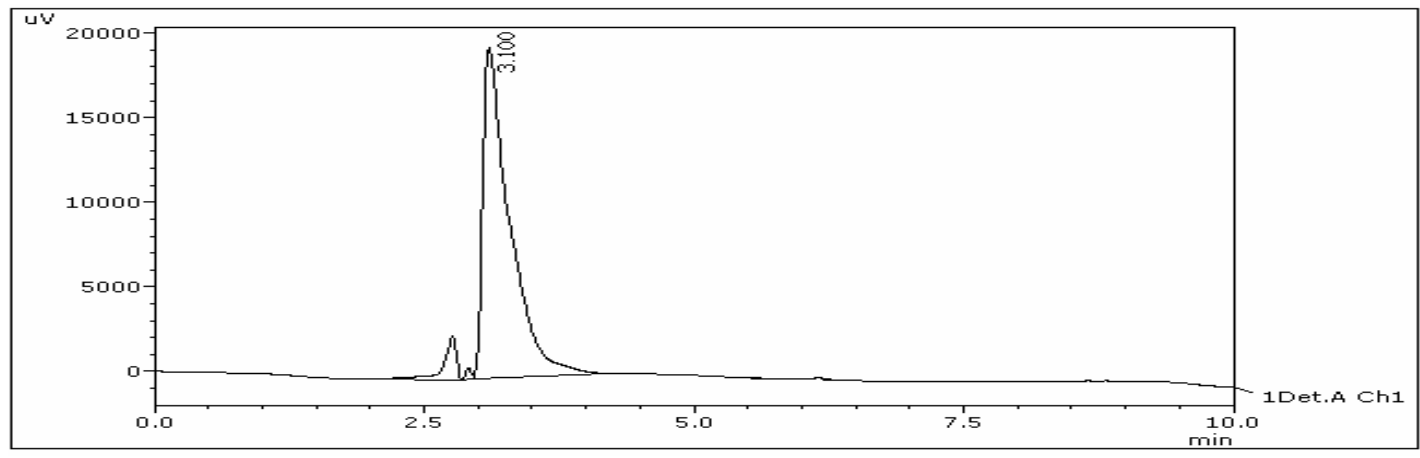

(a)

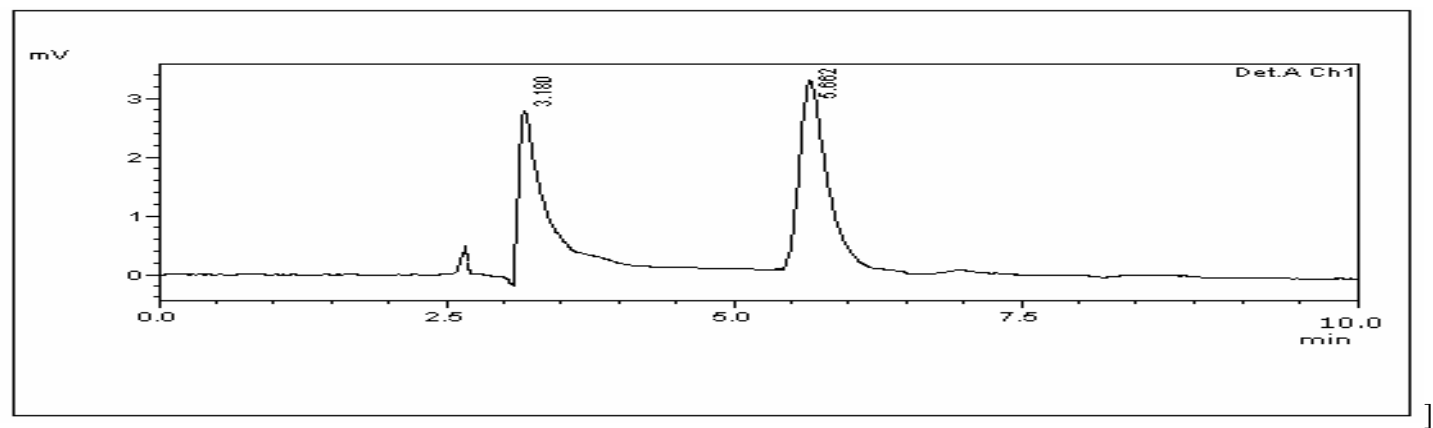

(b)

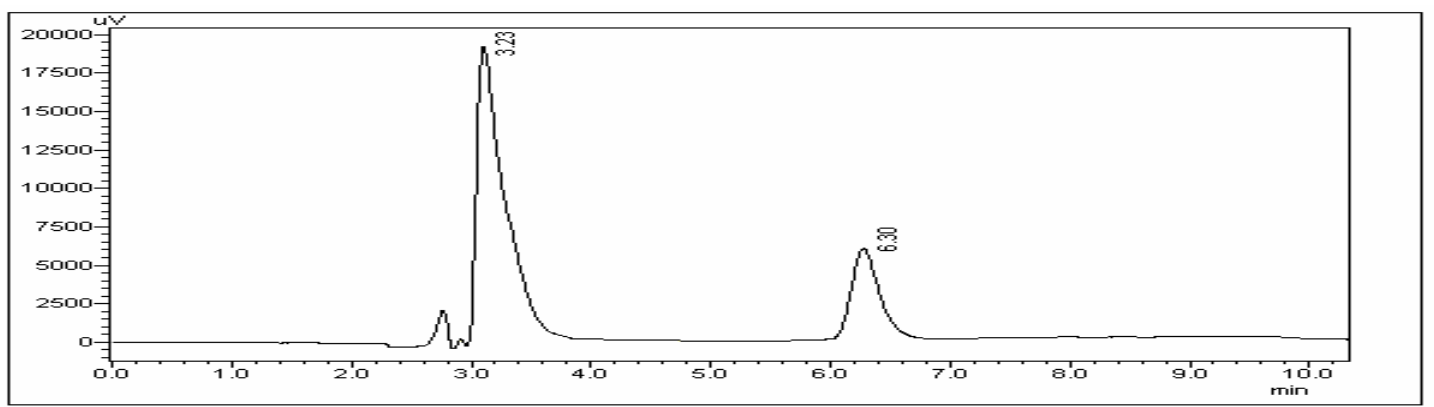

(c)

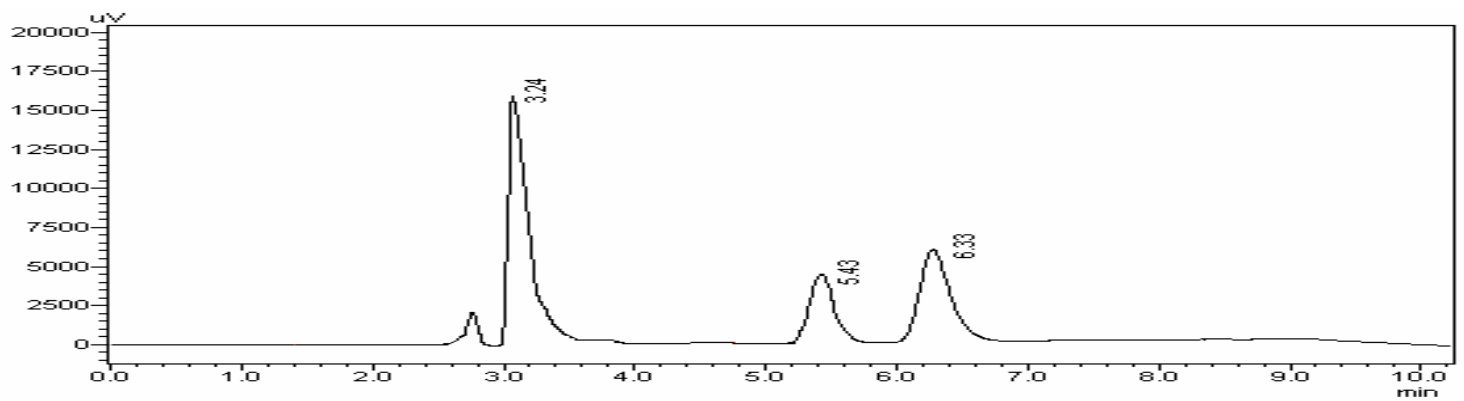

(d)

Figure 2. Representative chromatograms. a) Blank treated serum; b) Cefdinir in treated human serum; c) Cefaclor (I.S.) in treated human serum; d) Serum sample from a volunteer $3 \mathrm{hr}$ after administration of $300 \mathrm{mg}$ of cefdinir capsule. Peak at $5.4-5.6 \mathrm{~min}$ for cefdinir and $6.3 \mathrm{~min}$ for cefaclor. 
Linearity and range. The serum calibration curve was constructed with seven calibration samples $(0.05$ to $5 \mu \mathrm{g} / \mathrm{ml})$. The calibration curve was linear over the specified range. The mean \pm S.D. of the slope and intercept of the serum standards were $4.879 \pm$ 0.160 and $0.0719 \pm 0.020$, respectively. The coefficient of determination was greater than 0.996 on all calibration curves in serum.

Limit of quantification and limit of detection. The lower limit of quantification was defined as the lowest concentration on the calibration curve $(0.05$ $\mu \mathrm{g} / \mathrm{ml}$ ) for which an acceptable accuracy of $111.60 \%$ and a precision of $7.65 \%$ were obtained, while the minimum detectable quantity of cefdinir was found to be $0.02 \mu \mathrm{g} / \mathrm{ml}$.
Precision. The precision of the analytical method was well within the acceptable range of $15 \% \mathrm{CV}$ at all three points as presented in Table 1. The intra-day and inter-day $\% \mathrm{CV}$ at $0.05 \mu \mathrm{g} / \mathrm{ml}$ (lowest point of the calibration curve) were $7.65 \%$ and $9.72 \%$ respectively.

Accuracy. The percentage accuracy for estimation of cefdinir in serum was determined using standard addition method and was found to be well within the level of acceptance. The results are presented in Table 2.

Extraction efficiency (Recovery). The method of extraction of cefdinir was evaluated for efficiency and the results are shown in Table 3. The average recovery of cefdinir from serum was $96.43 \%$. The method showed good efficiency in terms of recovery.

Table 1. Intra-day and inter-day precision of cefdinir following oral administration of a single $300 \mathrm{mg}$ capsule formulation of cefdinir.

\begin{tabular}{|c|c|c|c|c|c|c|c|c|}
\hline \multicolumn{9}{|c|}{ Intra-day precision and accuracy ( $\mathrm{n}=5$ replicate samples) } \\
\hline \multicolumn{7}{|c|}{ Cefdinir concentrations $(\mu \mathrm{g} / \mathrm{ml})$} & \multirow{3}{*}{ Accuracy $(\%)$} & \multirow{3}{*}{$\mathrm{CV}(\%)$} \\
\hline \multirow{2}{*}{$\begin{array}{l}\text { Declared } \\
\text { conc. }\end{array}$} & \multicolumn{5}{|c|}{ Calculated concentration } & \multirow{2}{*}{ Mean (SD) } & & \\
\hline & 1 & 2 & 3 & 4 & 5 & & & \\
\hline 5.0 & 5.54 & 5.29 & 5.23 & 5.00 & 5.21 & $5.25(0.194)$ & 105.08 & 3.69 \\
\hline 0.5 & 0.53 & 0.51 & 0.46 & 0.47 & 0.48 & $0.49(0.029)$ & 98.00 & 5.95 \\
\hline 0.05 & 0.062 & 0.058 & 0.054 & 0.051 & 0.054 & $0.056(0.004)$ & 111.60 & 7.65 \\
\hline \multicolumn{9}{|c|}{ Inter-day precision and accuracy ( $\mathrm{n}=5$ days of replicate samples) } \\
\hline \multirow{3}{*}{$\begin{array}{l}\text { Declared } \\
\text { conc. }\end{array}$} & \multicolumn{6}{|c|}{ Cefdinir concentration $(\mu \mathrm{g} / \mathrm{ml})$} & \multirow{3}{*}{ Accuracy $(\%)$} & \multirow{3}{*}{$\mathrm{CV}(\%)$} \\
\hline & \multicolumn{5}{|c|}{ Calculated concentration } & \multirow{2}{*}{ Mean (SD) } & & \\
\hline & 1 & 2 & 3 & 4 & 5 & & & \\
\hline 5.0 & 5.25 & 5.17 & 4.89 & 4.53 & 4.8 & $4.93(0.291)$ & 98.56 & 5.90 \\
\hline 0.5 & 0.56 & 0.54 & 0.52 & 0.45 & 0.48 & $0.51(0.045)$ & 102.00 & 8.77 \\
\hline 0.05 & 0.051 & 0.047 & 0.045 & 0.039 & 0.048 & $0.05(0.004)$ & 92.00 & 9.72 \\
\hline
\end{tabular}

Table 2. Accuracy of the method for determining Cefdinir following oral administration of a single 300 mg capsule formulation of cefdinir.

\begin{tabular}{|c|c|c|c|c|}
\hline \multicolumn{3}{|c|}{ Concentration of cefdinir $(\mu \mathrm{g} / \mathrm{ml})$} & \multirow{2}{*}{$\begin{array}{l}\text { Total quantity of cefdinir } \\
\text { found }(\mu \mathrm{g} / \mathrm{ml}) \\
(\text { Mean } \pm \text { S.D. })(\mathrm{n}=4)\end{array}$} & \multirow{2}{*}{$\begin{array}{c}\% \text { Accuracy } \\
(\text { Mean } \pm \text { S.D. })(n=4)\end{array}$} \\
\hline Initial quantity (a) & $\begin{array}{l}\text { Quantity of standard } \\
\text { added (b) }\end{array}$ & Total quantity $(a+b)$ & & \\
\hline 0.1 & 0 & 0.1 & $0.11 \pm 0.015$ & $111.11 \pm 15.1$ \\
\hline 0.1 & 0.4 & 0.5 & $0.56 \pm 0.02$ & $112.22 \pm 4.9$ \\
\hline 0.5 & 0.5 & 1.0 & $0.91 \pm 0.02$ & $91.39 \pm 2.16$ \\
\hline 0.5 & 1.5 & 2.0 & $1.93 \pm 0.04$ & $96.28 \pm 1.90$ \\
\hline 1.0 & 1.0 & 2.0 & $2.01 \pm 0.02$ & $100.32 \pm 1.16$ \\
\hline 1.0 & 4.0 & 5.0 & $4.77 \pm 0.16$ & $95.37 \pm 3.23$ \\
\hline
\end{tabular}


Table 3. Serum cefdinir recovery following oral administration of a single $\mathbf{3 0 0} \mathbf{~ m g ~ c a p s u l e ~ f o r m u l a t i o n ~ o f ~ c e f d i n i r . ~}$

\begin{tabular}{|c|c|c|c|}
\hline \multicolumn{2}{|c|}{$\begin{array}{c}\text { Concentration of cefdinir } \\
(\mu \mathrm{g} / \mathrm{ml})\end{array}$} & \multirow[t]{2}{*}{ Recovery } & \multirow[t]{2}{*}{ Average } \\
\hline Added & Found & & \\
\hline 5 & 4.7474 & 94.948 & \\
\hline 0.5 & 0.50127 & 100.254 & 96.4256 \\
\hline 0.05 & 0.04704 & 94.0751 & \\
\hline
\end{tabular}

Table 4. Serum pharmacokinetic parameters of cefdinir following oral administration of a single $300 \mathrm{mg}$ capsule formulation of cefdinir.

\begin{tabular}{lccccccc}
\hline \multicolumn{1}{c}{ Pharmacokinetic parameters } & Mean & Median & Geometric Mean & SD & CV (\%) & Max & Min \\
\hline $\mathrm{C}_{\max }(\mu \mathrm{g} / \mathrm{ml})$ & 1.42 & 1.29 & 1.35 & 0.50 & 35.13 & 2.18 & 0.76 \\
$\mathrm{~T}_{\max }(\mathrm{hr})$ & 3.81 & 3.50 & 3.72 & 0.96 & 25.21 & 6.00 & 3.00 \\
$\mathrm{AUC}_{0-12}(\mathrm{hr}-\mu \mathrm{g} / \mathrm{ml})$ & 6.43 & 5.95 & 6.14 & 2.09 & 32.50 & 9.83 & 3.61 \\
$\mathrm{AUC}_{0-\infty}(\mathrm{hr}-\mu \mathrm{g} / \mathrm{ml})$ & 6.75 & 6.27 & 6.44 & 2.23 & 32.96 & 10.06 & 3.78 \\
$\mathrm{k}_{\mathrm{el}}\left(\mathrm{hr}^{-1}\right)$ & 0.36 & 0.33 & 0.35 & 0.08 & 22.52 & 0.49 & 0.25 \\
$\mathrm{AUMC}_{0-12}\left(\mathrm{hr}^{2}-\mu \mathrm{g} / \mathrm{ml}\right)$ & 30.33 & 29.30 & 28.85 & 10.03 & 33.07 & 46.41 & 16.39 \\
$\mathrm{AUMC}_{0-\infty}\left(\mathrm{hr}^{2}-\mu \mathrm{g} / \mathrm{ml}\right)$ & 35.24 & 34.49 & 33.33 & 12.49 & 35.45 & 56.85 & 18.88 \\
$\mathrm{t}_{1 / 2}(\mathrm{hr})$ & 2.03 & 2.10 & 1.99 & 0.44 & 21.80 & 2.79 & 1.40 \\
MRT $(\mathrm{hr})$ & 5.19 & 5.31 & 5.17 & 0.44 & 8.45 & 5.65 & 4.37 \\
\hline
\end{tabular}

Pharmacokinetic properties of cefdinir. The pharmacokinetic parameters of cefdinir are summarized in Table 4. The Mean (SD) $\mathrm{C}_{\max }$ of cefdinir was found to be $1.42(0.53) \mu \mathrm{g} / \mathrm{ml}$ attained at a mean $\mathrm{T}_{\max }$ of $3.81 \mathrm{hr}$. All volunteers presented an $\mathrm{AUC}_{0-\mathrm{t}} / \mathrm{AUC}_{0-\infty}$ ratio was greater than $80 \%$. The mean elimination half-life was $2.03 \mathrm{hrs}$. Mean serum drug concentrations of cefdinir for all the volunteers are presented in Figure 3.

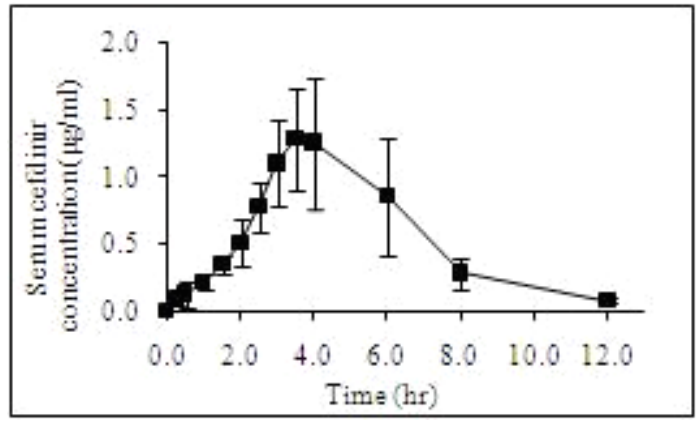

Figure 3. Mean (SD) cefdinir concentration-versus-time curve over 12 hours in adult healthy Bangladeshi volunteers $(\mathrm{N}=08)$.

A few HPLC-UV, LC-MS/MS, and GC-MS methods have been reported in different literatures. ${ }^{7,9}$ Some of these methods require complicated extraction instruments, long and tedious extraction procedures, and large amount of solvents or biological fluids for extraction while other methods have a long turnaround time during analysis. To minimize these limitations, the present investigation provides a rapid, selective and sensitive RP-HPLCUV method that has short and simple extraction procedure, consume small amount of solvents and biological fluid for extraction with a short turnaround time.

\section{CONCLUSION}

In the explored range, the present HPLC method was accurate, precise, and selective enough to allow the analysis of cefdinir in human serum after single oral dose of $300 \mathrm{mg}$ of cefdinir capsule which fulfils the acceptance criteria generally established for bioanalytical assays. The internal standard cefaclor, selected as structural analogues of cefdinir, was allowed to compensate the signal suppression effect and reduce inaccuracy problems. The present method offers an undoubted advantage in terms of overall analytical performance in comparison with the previously developed methods.

\section{REFERENCES}

1. Food and Drug Administration. 2007. Omnicef DN1235V2. http://www.accessdata.fda.gov/ drugsatfda_docs/ label/2008/ 050739s015,050749s021lbl.pdf pp.1-21 Accessed 15 Dec 2010 
2. Shah, P.B., Pundarikakshudu, K. 2006. Difference spectroscopic and reverse phase HPLC methods for the estimation of cefdinir in pharmaceutical dosage forms. Indian J Pharm Sci. 68, 90-93.

3. Physician's Desk Reference. 2003. 57th ed. [M], Montvale NJ: Thomson PDR, p. 489.

4. Bowlware, K.L., McCracken, G.H., Lozano-Hernandez, J., Ghaffar, F. 2006. Cefdinir pharmacokinetics and tolerability in children receiving $25 \mathrm{mg} / \mathrm{kg}$ once daily. Pediatr Infect Dis J. 25, 208-10.

5. Perry, C.M., Scott, L.J. 2004. Cefdinir: A review of its use in the management of mild-to-moderate bacterial infections. Drugs. 64, 1438-64.

6. Arguedas, A., Dagan, R., Leibovitz, E., Hoberman, A., Pichichero, M., Paris, M. 2006. A multicenter, open label, double tympanocentesis study of high dose cefdinir in children with acute otitis media at high risk of persistent or recurrent infection. Pediatr Infect Dis J. 25, 211-218.

7. Chen, Z., Zhang, J., Yu, J., Cao, G., Wu, X., Shi, Y. 2006. Selective method for the determination of cefdinir in human plasma using liquid chromatography electrospray ionization tandam mass spectrometry. J. Chromatography B, 834, 163169.

8. Shah, P. V., Midha, K. K., Findlay, W. A. J., Hill, M. H., Hulse, D. J., Mcgilveray, J. I. et al. 2000. Bioanalytical method validation-a revisit with a decade of progress. Pharmaceutical Res. 17, 1551-1557.
9. Hishida, A., Ohishi, K., Nagashima, S., Kanamaru, M., Obara, M., Kitada, A. 1998. Pharmacokinetic study of an oral cephalosporin, cefdinir, in hemodialysis patients. Antimicrob. Agents Chemother. 42, 1718-1724.

10. FDA Guidance for Industry. 2003. Bioavailability and Bioequivalence Studies for Orally Administered Drug Products. Rockville, MD: Office of Generic Drugs, Division of Bioequivalence, US Food and Drug Administration.

11. Anonymous. 2001. Guide for Industry, Bioanalytical Method Validation, U.S Department of Health and Human Services Food and Drug Administration (FDA).

12. World Medical Association Declaration of Helsinki: Ethical Principles for Medical Research Involving Human Subjects. 2000. Adopted by the 18th WMA General Assembly, Helsinki, Finland, June 1964, and amended by the 52nd WMA General Assembly, Edinburgh, Scotland. http://www.wma.net/e/policy/b3.htm Accessed Dec 15, 2010.

13. European Agency for the Evaluation of Medicinal Products, International Conference on Harmonization - World Health Organization. 2002. Guideline for Good Clinical Practice. ICH topic E6. Geneva, Switzerland: WHO. http://www.emea. europa.eu Accessed Dec 15, 2010.

14. Ullah, M.A., Shams-Ud-Dowla, Maruf, A.A., Azad, M.A., Shohag, M.H., Sultana, R., Latif, A.H., Hasnat, A. 2010. Relative bioavailability and pharmacokinetic properties of two different enteric formulations of esomeprazole in healthy Bangladeshi male volunteers: an open-label, single-dose, randomized-sequence, two-way crossover study. Clin Ther. 32, 1419-1426. 